\title{
ARRANGEMENTS AND LOCAL SYSTEMS
}

\author{
Daniel C. Cohen ${ }^{\dagger}$ And Peter Orlik ${ }^{\ddagger}$
}

\begin{abstract}
We use stratified Morse theory to construct a complex to compute the cohomology of the complement of a hyperplane arrangement with coefficients in a complex rank one local system. The linearization of this complex is shown to be the Aomoto complex of the arrangement. Using this result, we establish the relationship between the cohomology support loci of the complement and the resonance varieties of the Orlik-Solomon algebra for any arrangement, and show that the latter are unions of subspace arrangements in general, resolving a conjecture of Falk. We also obtain lower bounds for the local system Betti numbers in terms of those of the Orlik-Solomon algebra, recovering a result of Libgober and Yuzvinsky. For certain local systems, our results provide new combinatorial upper bounds on the local system Betti numbers. These upper bounds enable us to prove that in non-resonant systems the cohomology is concentrated in the top dimension, without using resolution of singularities.
\end{abstract}

\section{Introduction}

Let $\mathcal{A}=\left\{H_{1}, \ldots, H_{n}\right\}$ be a hyperplane arrangement in $\mathbb{C}^{\ell}$, with complement $M=M(\mathcal{A})=\mathbb{C}^{\ell} \backslash \bigcup_{j=1}^{n} H_{j}$. We assume that $\mathcal{A}$ contains $\ell$ linearly independent hyperplanes. Let $\lambda=\left(\lambda_{1}, \ldots, \lambda_{n}\right) \in \mathbb{C}^{n}$ be a collection of weights. Associated to $\lambda$, we have a rank one representation $\rho: \pi_{1}(M) \rightarrow \mathbb{C}^{*}$ given by $\gamma_{j} \mapsto t_{j}=\exp \left(2 \pi \mathrm{i} \lambda_{j}\right)$ for any meridian loop $\gamma_{j}$ about the hyperplane $H_{j}$ of $\mathcal{A}$, and a corresponding rank one local system $\mathcal{L}$ on $M$. The need to calculate the local system cohomology $H^{*}(M ; \mathcal{L})$ arises in several problems: the AomotoGelfand theory of multivariable hypergeometric integrals [AK, Ge]; representation theory of Lie algebras and quantum groups and solutions of the KnizhnikZamolodchikov differential equation in conformal field theory [Va]; determining the cohomology groups of the Milnor fiber of the non-isolated hypersurface singularity at the origin obtained by coning the arrangement [CS1].

Received July 19, 1999.

2000 Mathematics Subject Classification. 52C35, 55N25.

Key words and phrases. hyperplane arrangement, local system, Orlik-Solomon algebra, resonance variety, cohomology support locus.

$\dagger$ Partially supported by a grant from the Louisiana State University Council on Research and by grants LEQSF(1996-99)-RD-A-04 and LEQSF(1999-2002)-RD-A-01 from the Louisiana Board of Regents.

${ }^{\ddagger}$ Partially supported by the NSF. 
We call a system of weights $\lambda \in \mathbb{C}^{n}$ non-resonant if the Betti numbers of $M$ with coefficients in the associated local system $\mathcal{L}$ are minimal. The set of non-resonant weights is open and dense in $\mathbb{C}^{n}$.

Theorem 1.1. If $\lambda$ is non-resonant, then

$$
H^{q}(M ; \mathcal{L})=0 \text { for } q \neq \ell \text {, and } \operatorname{dim} H^{\ell}(M ; \mathcal{L})=|e(M)|,
$$

where $e(M)$ is the Euler characteristic of the complement.

We use the notation and results of [OT1]. Let $A=A(\mathcal{A})$ be the OrlikSolomon algebra of $\mathcal{A}$ generated by the 1 -dimensional classes $a_{j}, 1 \leq j \leq n$. It is the quotient of the exterior algebra generated by these classes by a homogeneous ideal, hence a finite dimensional graded $\mathbb{C}$-algebra. There is an isomorphism of graded algebras $H^{*}(M ; \mathbb{C}) \simeq A(\mathcal{A})$. In particular, $\operatorname{dim} A^{q}(\mathcal{A})=b_{q}(\mathcal{A})$ where $b_{q}(\mathcal{A})=\operatorname{dim} H^{q}(M ; \mathbb{C})$ denotes the $q$-th Betti number of $M$ with trivial local coefficients $\mathbb{C}$.

Theorem 1.1 is a consequence of two results which are quite different in nature. One is the work of Esnault-Schechtman-Viehweg [ESV], refined by Schechtman-Terao-Varchenko [STV], and obtained by using Deligne's work [De]. It involves resolution of singularities and techniques of algebraic geometry. Here the de Rham complex with a twisted differential is used to compute $H^{*}(M ; \mathcal{L})$, and the main aim is to reduce the infinite dimensional cochain groups to a complex with finite dimensional cochain groups. In the passage from consideration of global rational differential forms in $\mathbb{C}^{\ell}$ with poles of arbitrary order on the hyperplanes of $\mathcal{A}$ to poles of order one (logarithmic), certain geometric conditions on $\lambda$ arise. The final result is that for suitable $\lambda$ there is an isomorphism

$$
H^{q}(M ; \mathcal{L}) \simeq H^{q}\left(A^{\bullet}, a_{\lambda} \wedge\right),
$$

where the Orlik-Solomon algebra with differential $A^{q} \rightarrow A^{q+1}$ given by multiplication by $a_{\lambda}=\sum_{j=1}^{n} \lambda_{j} a_{j}$ is identified with a subcomplex of the twisted de Rham complex. The second result, due to Yuzvinsky [Yu], is purely combinatorial in nature. It shows that if $\lambda$ satisfies certain combinatorial conditions, then

$$
H^{q}\left(A^{\bullet}, a_{\lambda} \wedge\right)=0 \text { for } q \neq \ell, \text { and } \operatorname{dim} H^{\ell}\left(A^{\bullet}, a_{\lambda} \wedge\right)=\operatorname{dim} H^{\ell}(M ; \mathcal{L})=|e(M)| .
$$

The geometric conditions on $\lambda$ noted above arise from conditions on the monodromy of the local system about certain intersections of hyperplanes of $\mathcal{A}$. These conditions derive from Aomoto's 1973 work [Ao], where slightly stronger monodromy conditions were considered. Indeed, the results of [ESV] mentioned previously resolved a conjecture from this paper. Kohno [Ko] used similar (strong) monodromy conditions to prove a vanishing theorem analogous to Theorem 1.1 for local system cohomology and locally finite homology, and exhibited a basis for the non-zero homology group in a special case. Hattori [Ha] showed that for general position arrangements only the trivial local system is resonant. More recently, Falk-Terao [FT] constructed a basis for the non-zero local system 
cohomology group in the non-resonant case for any arrangement. Thus, much is known in the case of non-resonant weights. See [OT2] for a detailed discussion.

Substantially less is known about resonant weights, which are of particular interest in several of the applications noted above. This interest has been a motivating factor in much recent work, including [C3, CS1, CS4, Fa, L1, LY]. In this paper, the local system cohomology is studied using a complex to compute $H^{*}(M ; \mathcal{L})$ which arises from stratified Morse theory. The terms of this complex are finite dimensional but the boundary maps are not easily computed. In fact, we construct a universal complex, $\left(K_{\Lambda}^{\bullet}(\mathcal{A}), \Delta^{\bullet}(x)\right)$, where $x=\left(x_{1}, \ldots, x_{n}\right)$ are non-zero complex variables, with the property that the specialization $x_{j} \mapsto t_{j}$ calculates $H^{*}(M ; \mathcal{L})$. There is a similar universal complex, called the Aomoto complex $\left(A_{R}^{\bullet}(\mathcal{A}), a_{y} \wedge\right)$, where $y=\left(y_{1}, \ldots, y_{n}\right)$ are variables, with the property that the specialization $y_{j} \mapsto \lambda_{j}$ calculates $H^{*}\left(A^{\bullet}, a_{\lambda} \wedge\right)$. Our first result is:

Theorem 1.2. For any arrangement $\mathcal{A}$, the Aomoto complex $\left(A_{R}^{\bullet}(\mathcal{A}), a_{y} \wedge\right)$ is chain equivalent to the linearization of the universal complex $\left(K_{\Lambda}^{\bullet}(\mathcal{A}), \Delta^{\bullet}(x)\right)$.

Since this theorem applies to all weight systems, we obtain corollaries for resonant weights. One is a proof of Falk's conjecture that the resonance varieties of the Orlik-Solomon algebra are unions of subspace arrangements, see Corollary 3.8. Another is the known lower bound of Proposition 3.2:

$$
\operatorname{dim} H^{q}\left(A^{\bullet}, a_{\lambda} \wedge\right) \leq \operatorname{dim} H^{q}(M ; \mathcal{L}) .
$$

In the other direction, the upper bounds

$$
\operatorname{dim} H^{q}(M ; \mathcal{L}) \leq \operatorname{dim} H^{q}(M ; \mathbb{C})
$$

were conjectured in $[\mathrm{AK}]$, and established in [C2]. In most applications where resonant weights occur, the $\lambda_{j}$ are rational numbers. We call the associated local system rational. Our next aim is to obtain better upper bounds for $\operatorname{dim} H^{q}(M ; \mathcal{L})$ for rational local systems. Let $\lambda_{j}=k_{j} / N$, where $k_{j} \in \mathbb{Z}$ and $N \in \mathbb{N}$. We define a complex, $\left(A_{N}^{\bullet}, \bar{a}_{k} \wedge\right)$, whose cochain groups are the graded parts of the Orlik-Solomon algebra with coefficients in the ring $\mathbb{Z}_{N}=\mathbb{Z} / N \mathbb{Z}$, and whose differential operator is obtained from $a_{k}=\sum_{j=1}^{n} k_{j} a_{j}$ by reduction $\bmod N$, and prove:

Theorem 1.3. Let $\lambda=k / N$ be a system of rational weights, and let $\mathcal{L}$ be the associated rational local system on the complement $M$ of $\mathcal{A}$. Then, for each $q$,

$$
\operatorname{dim}_{\mathbb{C}} H^{q}(M ; \mathcal{L}) \leq \operatorname{rank}_{\mathbb{Z}_{N}} H^{q}\left(A_{N}^{\bullet}, \bar{a}_{k} \wedge\right) .
$$

This result about rational, and often resonant, weights is used to give a proof of Theorem 1.1 (about non-resonant weights) which does not rely on resolution of singularities. Examples show that the inequalities in both the upper and lower bounds may be strict. 


\section{Linear approximation}

Let $\mathcal{A}=\left\{H_{1}, \ldots, H_{n}\right\}$ be a hyperplane arrangement in $\mathbb{C}^{\ell}$, and let $\mathcal{L}$ be a complex rank one local system on the complement $M$ of $\mathcal{A}$. In this section, we show that the Orlik-Solomon algebra complex $\left(A^{\bullet}(\mathcal{A}), a_{\lambda} \wedge\right)$ is, in a natural sense, a linear approximation of a Morse theoretic complex, $\left(K^{\bullet}(\mathcal{A}), \Delta^{\bullet}\right)$, the cohomology of which is isomorphic to that of $M$ with coefficients in $\mathcal{L}$.

2.1. Preliminaries. Let $\mathcal{B}$ denote the Boolean arrangement in $\mathbb{C}^{n}$, with coordinates $z_{1}, \ldots, z_{n}$. A defining polynomial for $\mathcal{B}$ is given by $Q(\mathcal{B})=z_{1} \cdots z_{n}$, and the complement of $\mathcal{B}$ is the complex $n$-torus, $T=\left(\mathbb{C}^{*}\right)^{n}$.

Proposition 2.2. Let $\mathcal{A}$ be an arrangement of $n$ hyperplanes in $\mathbb{C}^{\ell}$. Then the complement $M$ of $\mathcal{A}$ may be realized as a linear section of the complex $n$-torus $T$.

Proof. Without loss of generality, assume that $\mathcal{A}$ is an essential arrangement in $\mathbb{C}^{\ell}$, so that $\mathcal{A}$ contains $\ell$ independent hyperplanes. Choose coordinates $z_{1}, \ldots, z_{\ell}$ for $\mathbb{C}^{\ell}$. Ordering the hyperplanes of $\mathcal{A}$ appropriately, a defining polynomial for $\mathcal{A}$ is then given by $Q(\mathcal{A})=z_{1} \cdots z_{\ell} \cdot \alpha_{\ell+1} \cdots \alpha_{n}$, where $\alpha_{j}=\alpha_{j}\left(z_{1}, \ldots, z_{\ell}\right)$ is a linear polynomial.

Let $S$ denote the $\ell$-dimensional affine subspace of $\mathbb{C}^{n}$ defined by the equations $\left\{z_{j}=\alpha_{j}\left(z_{1}, \ldots, z_{\ell}\right) \mid \ell+1 \leq j \leq n\right\}$. Identifying $\mathbb{C}^{\ell}$ with $S$ in the obvious manner, it is readily checked that $M=S \cap T$.

Let $\lambda=\left(\lambda_{1}, \ldots, \lambda_{n}\right) \in \mathbb{C}^{n}$ be a collection of weights. Associated to $\lambda$, we have a rank one representation $\rho: \pi_{1}(M) \rightarrow \mathbb{C}^{*}$ given by $\gamma_{j} \mapsto t_{j}=\exp \left(2 \pi \mathrm{i} \lambda_{j}\right)$ for any meridian loop $\gamma_{j}$ about the hyperplane $H_{j}$ of $\mathcal{A}$, and a corresponding rank one local system $\mathcal{L}$ on $M$. Note that the representation $\rho$ factors through the first homology $H_{1}(M ; \mathbb{Z})$, which is generated by the classes $\left[\gamma_{j}\right]$. Since these classes also generate $H_{1}(T ; \mathbb{Z})=\pi_{1}(T)$, the local system $\mathcal{L}$ extends to a local system on $T$, which we continue to denote by $\mathcal{L}$.

2.3. The Morse theoretic complex. For any complex local system $\mathcal{L}$ on the complement of an arrangement $\mathcal{A}$, in [C1] we used stratified Morse theory to construct a complex $\left(K^{\bullet}(\mathcal{A}), \Delta^{\bullet}\right)$, the cohomology of which is naturally isomorphic to $H^{*}(M ; \mathcal{L})$, the cohomology of $M$ with coefficients in $\mathcal{L}$. We give a brief description of this complex (for a rank one local system) and record some relevant results from $[\mathrm{C} 1]$.

An edge of $\mathcal{A}$ is a nonempty intersection of hyperplanes. The arrangement $\mathcal{A}$ determines a Whitney stratification of $S=\mathbb{C}^{\ell}$, with a stratum, $S_{X}=X \backslash$ $\bigcup_{Y \subset X} Y$, of codimension $p$ associated to each codimension $p$ edge $X$ of $\mathcal{A}$. (The stratum $S_{X}$ is given by $M\left(\mathcal{A}^{X}\right)$, see [OT1].) Let $\emptyset=F_{-1} \subset F_{0} \subset F_{1} \subset F_{2} \subset$ $\cdots \subset F_{\ell}=S$ be a flag in $S$ which is transverse to the stratification determined by $\mathcal{A}$, so that $\operatorname{dim} F_{q} \cap S_{X}=q-\operatorname{codim} S_{X}$ for each stratum, where a negative dimension indicates that $F_{q} \cap S_{X}=\emptyset$. Such a flag may be constructed using a Morse function on $S$ that is weakly self-indexing with respect to the above stratification, see [C1, Section 1]. 
The sets $M_{q}=F_{q} \cap M$ form a filtration, $\emptyset=M_{-1} \subset M_{0} \subset M_{1} \subset \cdots \subset$ $M_{\ell}=M$. By construction, for each $q$, the closure of $M_{q}$ intersects all strata of codimension at most $q$, and intersects no stratum of codimension greater than $q$. This filtration is well-suited for the study of local system cohomology in the sense of the following.

Proposition 2.4. For each $q, 0 \leq q \leq \ell$, we have $H^{i}\left(M_{q}, M_{q-1} ; \mathcal{L}\right)=0$ if $i \neq q$, and $\operatorname{dim} H^{q}\left(M_{q}, M_{q-1} ; \mathcal{L}\right)=b_{q}(\mathcal{A})$.

This Proposition may be proved using stratified Morse theory [GM]. For details, the reader is referred to [C1, Sections 2, 3, and 5].

For each $q$, let $K^{q}(\mathcal{A})=H^{q}\left(M_{q}, M_{q-1} ; \mathcal{L}\right)$ and then denote by $\Delta^{q}$ the boundary homomorphism $H^{q}\left(M_{q}, M_{q-1} ; \mathcal{L}\right) \rightarrow H^{q+1}\left(M_{q+1}, M_{q} ; \mathcal{L}\right)$ of the triple $\left(M_{q+1}, M_{q}, M_{q-1}\right)$. It is readily checked that the composition $\Delta^{q+1} \circ \Delta^{q}=0$. Thus the system of complex vector spaces and linear maps $\left(K^{\bullet}(\mathcal{A}), \Delta^{\bullet}\right)$ is a complex. The following is a special case of [C1, Theorem 2.4].

Theorem 2.5. The cohomology of the complex $\left(K^{\bullet}(\mathcal{A}), \Delta^{\bullet}\right)$ is naturally isomorphic to $H^{*}(M ; \mathcal{L})$, the cohomology of $M$ with coefficients in the local system $\mathcal{L}$.

Remark 2.6. As shown in [C2], the inequalities $\operatorname{dim} H^{q}(M ; \mathcal{L}) \leq \operatorname{dim} H^{q}(M ; \mathbb{C})$ noted in the Introduction follow immediately from this result. See also [Ma].

Example 2.7. We discuss first the important special case of the torus. Recall that $\mathcal{B}$ denotes the Boolean arrangement, consisting of the coordinate hyperplanes in $\mathbb{C}^{n}$, and that a rank one local system $\mathcal{L}$ on the complement of any arrangement of $n$ hyperplanes extends naturally to a local system on the torus $T=\left(\mathbb{C}^{*}\right)^{n}$, the complement of $\mathcal{B}$. For this arrangement (and more generally any general position arrangement), the Morse theoretic complex constructed above admits a complete description, see [C1, Section 7$]$ for details. Denote this complex by $\left(K^{\bullet}(\mathcal{B}), D^{\bullet}\right)$.

By Proposition 2.4 above, the terms of this complex are vector spaces of dimension $\operatorname{dim} K^{q}(\mathcal{B})=\operatorname{dim} H^{q}(T ; \mathbb{C})=b_{q}(\mathcal{B})=\left(\begin{array}{l}n \\ q\end{array}\right)$. Thus, as a graded group, the complex $K^{\bullet}(\mathcal{B})$ may be identified with the cohomology of $T$, which in turn is isomorphic to the exterior algebra $E=\bigwedge E^{1}$, where $E^{1}=\oplus_{j=1}^{n} \mathbb{C} e_{j}$.

The local system $\mathcal{L}$ is induced by the representation $\rho: \pi_{1}(T) \rightarrow \mathbb{C}^{*}$ defined by $\gamma_{j} \mapsto t_{j}$, where $\gamma_{j}$ is a meridian loop about the hyperplane $H_{j}$ and then $t_{j}=\exp \left(2 \pi \mathrm{i} \lambda_{j}\right)$. Under the identification $K^{\bullet}(\mathcal{B})=E$ above, the boundary $\operatorname{map} D^{q}: K^{q}(\mathcal{B}) \rightarrow K^{q+1}(\mathcal{B})$ is given by

$$
D^{q}\left(e_{J}\right)=e_{t} \wedge e_{J}=\left(\left(t_{1}-1\right) e_{1}+\cdots+\left(t_{n}-1\right) e_{n}\right) \wedge e_{J},
$$

where $e_{J}=e_{j_{1}} \wedge \cdots \wedge e_{j_{q}}$ if $J=\left\{j_{1}, \ldots, j_{q}\right\}$.

2.8. Approximation. We now return to an arbitrary arrangement $\mathcal{A}$ of $n$ hyperplanes and show that the Morse theoretic complex described above is approximated by the Orlik-Solomon algebra complex. Let $\lambda=\left(\lambda_{1}, \ldots, \lambda_{n}\right)$ be a weight 
vector in $\mathbb{C}^{n}, t=\left(t_{1}, \ldots, t_{n}\right)$ the associated point in the complex torus $\left(\mathbb{C}^{*}\right)^{n}$, and $\mathcal{L}$ the corresponding local system on $M(\mathcal{A})$. It is important to note that if $m=\left(m_{1}, \ldots, m_{n}\right)$ is a tuple of integers, then $\lambda+m=\left(\lambda_{1}+m_{1}, \ldots, \lambda_{n}+m_{n}\right)$ determines the same $t$ and $\mathcal{L}$.

As evidenced by Proposition 2.4, the dimensions of the terms of the complex $\left(K^{\bullet}(\mathcal{A}), \Delta^{\bullet}\right)$ are independent of $t$ (resp., $\left.\lambda, \mathcal{L}\right)$. To indicate the dependence of the complex on $t$, we write $\mathcal{L}=\mathcal{L}_{t}, \Delta^{\bullet}=\Delta^{\bullet}(t)$, and view these boundary maps as functions of $t$. These observations may be formalized as follows. Let $\Lambda=$ $\mathbb{C}\left[x_{1}^{ \pm 1}, \ldots, x_{n}^{ \pm 1}\right]$ denote the ring of complex Laurent polynomials in $n$ commuting variables, and for each $q$, let $K_{\Lambda}^{q}(\mathcal{A})=\Lambda \otimes_{\mathbb{C}} K^{q}(\mathcal{A})$.

Theorem 2.9. Given an arrangement $\mathcal{A}$ of $n$ hyperplanes with complement $M$, there exists a universal complex $\left(K_{\Lambda}^{\bullet}(\mathcal{A}), \Delta(x)\right)$ with the following properties:

1. The terms are free $\Lambda$-modules, whose ranks are given by the Betti numbers of $M, K_{\Lambda}^{q}(\mathcal{A}) \simeq \Lambda^{b_{q}(\mathcal{A})}$.

2. The boundary maps, $\Delta^{q}(x): K_{\Lambda}^{q}(\mathcal{A}) \rightarrow K_{\Lambda}^{q+1}(\mathcal{A})$ are $\Lambda$-linear.

3. For each $t \in\left(\mathbb{C}^{*}\right)^{n}$, the specialization $x \mapsto t$ yields the complex $\left(K^{\bullet}(\mathcal{A}), \Delta^{\bullet}(t)\right)$, the cohomology of which is isomorphic to $H^{*}\left(M ; \mathcal{L}_{t}\right)$, the cohomology of $M$ with coefficients in the local system associated to $t$.

Remark 2.10. For the Boolean arrangement $\mathcal{B}$ of $n$ hyperplanes, the universal complex $\left(K_{\Lambda}^{\bullet}(\mathcal{B}), D^{\bullet}(x)\right)$ is dual to the standard free $\Lambda \simeq \mathbb{Z} \mathbb{Z}^{n}$-resolution of the integers. This follows from the description of the complex $\left(K^{\bullet}(\mathcal{B}), D^{\bullet}(t)\right)$ given in Example 2.7.

Proposition 2.11. Let $\mathcal{A}$ be an arrangement of $n$ hyperplanes in $\mathbb{C}^{\ell}$. Then there exists a chain map $\Psi^{\bullet}(x): K_{\Lambda}^{\bullet}(\mathcal{B}) \rightarrow K_{\Lambda}^{\bullet}(\mathcal{A})$ from the universal complex of the Boolean arrangement $\mathcal{B}$ to the universal complex of $\mathcal{A}$.

Proof. Recall from Proposition 2.2 that we realize the complement of $\mathcal{A}$ as an $\ell$-dimensional linear section, $M=S \cap T$, of the complement of $\mathcal{B}$, and that any rank one local system $\mathcal{L}$ on $M$ extends to a local system on $T$.

For such a local system, the complexes $K^{\bullet}(\mathcal{A})$ and $K^{\bullet}(\mathcal{B})$ are constructed using flags $\emptyset=F_{-1} \subset F_{0} \subset F_{1} \subset \cdots \subset F_{\ell}=S$ and $\emptyset=F_{-1}^{\prime} \subset F_{0}^{\prime} \subset F_{1}^{\prime} \subset$ $\cdots \subset F_{n}^{\prime}=\mathbb{C}^{n}$ respectively. Since the affine subspace $S$ is transverse to the hyperplanes of $\mathcal{B}$, we may assume that $F_{q}^{\prime}=F_{q}$ for $q \leq 1$. Thus the inclusion $M \cap F_{q} \subseteq T \cap F_{q}$ induces canonical isomorphisms $\psi^{q}: K^{q}(\mathcal{B}) \stackrel{\sim}{\longrightarrow} K^{q}(\mathcal{A})$ for $q \leq 1$, and for every $t \in\left(\mathbb{C}^{*}\right)^{n}$ we have $\Delta^{0}(t)=D^{0}(t)$. This yields isomorphisms $\Psi^{q}=\mathrm{id} \otimes \psi^{q}: K_{\Lambda}^{q}(\mathcal{B}) \stackrel{\sim}{\longrightarrow} K_{\Lambda}^{q}(\mathcal{A})$ for $q \leq 1$, and we have $\Delta^{0}(x)=D^{0}(x)$.

Now, as noted in Remark 2.10, the complex $\left(K_{\Lambda}^{\bullet}(\mathcal{B}), D^{\bullet}(x)\right)$ is dual to the standard $\Lambda$-resolution of $\mathbb{Z}$, which is of course acyclic. Thus it follows from the acyclic models theorem that there is a chain map $\Psi^{\bullet}(x): K_{\Lambda}^{\bullet}(\mathcal{B}) \rightarrow K_{\Lambda}^{\bullet}(\mathcal{A})$ covering $\Psi^{q}(q \leq 1)$.

As is the case for the local system cohomology of the complement, there is a universal complex for the cohomology, $H^{*}\left(A^{\bullet}(\mathcal{A}), a_{\lambda} \wedge\right)$, of the Orlik-Solomon 
algebra. Let $R=\mathbb{C}\left[y_{1}, \ldots, y_{n}\right]$ be the polynomial ring. The Aomoto complex $\left(A_{R}^{\bullet}(\mathcal{A}), a_{y} \wedge\right)$ has terms $A_{R}^{q}(\mathcal{A})=R \otimes_{\mathbb{C}} A^{q}(\mathcal{A})$, and boundary maps given by $p(y) \otimes \eta \mapsto \sum y_{j} p(y) \otimes a_{j} \wedge \eta$. For $\lambda \in \mathbb{C}^{n}$, the specialization $y \mapsto \lambda$ of the Aomoto complex $\left(A_{R}^{\bullet}(\mathcal{A}), a_{y} \wedge\right)$ yields the Orlik-Solomon algebra complex $\left(A^{\bullet}(\mathcal{A}), a_{\lambda} \wedge\right)$.

Fix a basis for the Orlik-Solomon algebra of $\mathcal{A}$. Evidently, this yields a basis for each term $A_{R}^{q}(\mathcal{A})$ of the Aomoto complex. Let $\mu^{q}(y)$ denote the matrix of $a_{y} \wedge: A_{R}^{q}(\mathcal{A}) \rightarrow A_{R}^{q+1}(\mathcal{A})$ with respect to this basis.

Lemma 2.12. For each $q$, the entries of $\mu^{q}(y)$ are integral linear forms in $y_{1}, \ldots, y_{n}$.

Proof. First note that this holds in the case where $\mathcal{A}=\mathcal{B}$ is the Boolean arrangement. In this case, the Orlik-Solomon algebra is the exterior algebra $E$, and the boundary maps of the Aomoto complex are given by $e_{J} \mapsto e_{y} \wedge e_{J}=$ $\sum_{j=1}^{n} y_{j} \otimes e_{j} \wedge e_{J}$ on generators.

For an arbitrary arrangement $\mathcal{A}$, the Aomoto complex $\left(A_{R}^{\bullet}(\mathcal{A}), a_{y} \wedge\right)$ may be realized as the quotient of the Aomoto complex $\left(A_{R}^{\bullet}(\mathcal{B}), e_{y} \wedge\right)$ of the Boolean arrangement by the subcomplex $\left(I_{R}^{\bullet}(\mathcal{A}), e_{y} \wedge\right)$, where $I_{R}^{\bullet}(\mathcal{A})$ denotes the tensor product of $R$ with the Orlik-Solomon ideal $I(\mathcal{A})$. Since the ideal $I(\mathcal{A})$ is defined by integral linear combinations of the generators of the exterior algebra, the result follows.

The main result of this section is the following.

Theorem 2.13. For any arrangement $\mathcal{A}$, the Aomoto complex $\left(A_{R}^{\bullet}(\mathcal{A}), a_{y} \wedge\right)$ is chain equivalent to the linearization of the universal complex $\left(K_{\Lambda}^{\bullet}(\mathcal{A}), \Delta^{\bullet}(x)\right)$

Proof. For each $q$, fix a basis for $K_{\Lambda}^{q}(\mathcal{A})$. Since $\operatorname{rank}_{\Lambda} K_{\Lambda}^{q}(\mathcal{A})=\operatorname{dim}_{\mathbb{C}} A^{q}(\mathcal{A})=$ $b_{q}(\mathcal{A})$, the basis for $K_{\Lambda}^{\bullet}(\mathcal{A})$ may be chosen in one-to-one correspondence with that of $A(\mathcal{A})$. We shall not distinguish between the boundary map, $\Delta^{q}(x)$ : $K_{\Lambda}^{q}(\mathcal{A}) \rightarrow K_{\Lambda}^{q+1}(\mathcal{A})$, of the universal complex and its matrix with respect to the chosen basis.

The entries of $\Delta^{q}(x)$ are elements of the Laurent polynomial ring $\Lambda$, the coordinate ring of the complex algebraic $n$-torus. Via the specialization $x \mapsto t \in$ $\left(\mathbb{C}^{*}\right)^{n}$, we shall view them as holomorphic functions $\left(\mathbb{C}^{*}\right)^{n} \rightarrow \mathbb{C}$. Similarly, for each $q$, we view $\Delta^{q}$ as a holomorphic map $\Delta^{q}:\left(\mathbb{C}^{*}\right)^{n} \rightarrow \operatorname{Mat}(\mathbb{C})$ by $t \mapsto \Delta^{q}(t)$.

Let $\mathbf{1}=(1, \ldots, 1)$ denote the identity element of $\left(\mathbb{C}^{*}\right)^{n}$. The holomorphic tangent space of the complex $n$-torus at $\mathbf{1}$ is $\mathrm{T}_{\mathbf{1}}\left(\mathbb{C}^{*}\right)^{n}=\mathbb{C}^{n}$. Identify the coordinates $y=\left(y_{1}, \ldots, y_{n}\right)$ of this tangent space with the variables appearing in the Aomoto complex. The exponential map $\mathrm{T}_{\mathbf{1}}\left(\mathbb{C}^{*}\right)^{n} \rightarrow\left(\mathbb{C}^{*}\right)^{n}$ is induced by $\exp : \mathbb{C} \rightarrow \mathbb{C}^{*}, y_{j} \mapsto e^{y_{j}}=x_{j}$.

The specialization $x \mapsto \mathbf{1}$ corresponding to the trivial local system yields a complex $\left(K^{\bullet}(\mathcal{A}), \Delta^{\bullet}(\mathbf{1})\right)$ with trivial boundary maps, $\Delta^{q}(\mathbf{1})=0$ for each $q$. This follows from Proposition 2.4. See [GM, III.3] for a detailed discussion of this phenomenon in homology.

If $f \in \Lambda$ is an entry of $\Delta^{q}(x)$, then the map $f:\left(\mathbb{C}^{*}\right)^{n} \rightarrow \mathbb{C}$ satisfies $f(\mathbf{1})=0$. For such a Laurent polynomial (resp., map), the derivative at the identity, $f_{*}$ : 
$\mathrm{T}_{\mathbf{1}}\left(\mathbb{C}^{*}\right)^{n}=\mathbb{C}^{n} \rightarrow \mathbb{C}=\mathrm{T}_{0} \mathbb{C}$, is given by

$$
f_{*}(y)=\left.\frac{d}{d s} f\left(e^{s y_{1}}, \ldots, e^{s y_{n}}\right)\right|_{s=0} .
$$

Similarly, for $F \in \operatorname{Mat}(\Lambda)$ satisfying $F(\mathbf{1})=0$, we have $F_{*}: \mathrm{T}_{\mathbf{1}}\left(\mathbb{C}^{*}\right)^{n} \rightarrow$ $\mathrm{T}_{0} \operatorname{Mat}(\mathbb{C})=\operatorname{Mat}(\mathbb{C})$. For two Laurent polynomials $f$ and $g$, the product rule yields $(f g)_{*}(y)=f_{*}(y) g(\mathbf{1})+f(\mathbf{1}) g_{*}(y)$. More generally, for $F \in \operatorname{Mat}_{p \times q}(\Lambda)$ and $G \in \operatorname{Mat}_{q \times r}(\Lambda)$, using matrix multiplication and the differentiation rules we have

$$
(F G)_{*}(y)=F_{*}(y) \cdot G(\mathbf{1})+F(\mathbf{1}) \cdot G_{*}(y) .
$$

Now recall the chain map $\Psi^{\bullet}(x): K_{\Lambda}^{\bullet}(B) \rightarrow K_{\Lambda}^{\bullet}(\mathcal{A})$ from the universal complex of the Boolean arrangement $\mathcal{B}$ to that of $\mathcal{A}$ described in Proposition 2.11. As is the case for the boundary maps $D^{q}(x)$ and $\Delta^{q}(x)$ of the two complexes, we view $\Psi^{q}(x)$ as a holomorphic map $\left(\mathbb{C}^{*}\right)^{n} \rightarrow \operatorname{Mat}(\mathbb{C})$, and we do not distinguish between this map and its matrix. Since $\Psi^{\bullet}(x)$ is a chain map, we have $D^{q}(x) \cdot \Psi^{q+1}(x)=\Psi^{q}(x) \cdot \Delta^{q}(x)$ for each $q$. We differentiate at $\mathbf{1}$ using the product rule (1) above to obtain

$$
D_{*}^{q}(y) \cdot \Psi^{q+1}(\mathbf{1})+D^{q}(\mathbf{1}) \cdot \Psi_{*}^{q+1}(y)=\Psi_{*}^{q}(y) \cdot \Delta^{q}(\mathbf{1})+\Psi^{q}(\mathbf{1}) \cdot \Delta_{*}^{q}(y) .
$$

Since $D^{q}(\mathbf{1})=0$ and $\Delta^{q}(\mathbf{1})=0$ for all $q$, we thus have

$$
D_{*}^{q}(y) \cdot \Psi^{q+1}(\mathbf{1})=\Psi^{q}(\mathbf{1}) \cdot \Delta_{*}^{q}(y) .
$$

Recall that $R=\mathbb{C}\left[y_{1}, \ldots, y_{n}\right]$ denotes the polynomial ring. For $F \in \operatorname{Mat}(\Lambda)$, view the derivative $F_{*}(y)$ as a linear map between free $R$-modules in the obvious way. Consider the systems of free $R$-modules and $R$-linear maps

$$
\left(K_{R}^{\bullet}(\mathcal{A}), \Delta_{*}^{\bullet}(y)\right) \quad \text { and } \quad\left(K_{R}^{\bullet}(\mathcal{B}), D_{*}^{\bullet}(y)\right)
$$

where $K_{R}^{q}(\mathcal{A})=R \otimes_{\mathbb{C}} K^{q}(\mathcal{A})$ and $K_{R}^{q}(\mathcal{B})=R \otimes_{\mathbb{C}} K^{q}(\mathcal{B})$. From the description of the universal complex of the Boolean arrangement stemming from Example 2.7 and the fact that $\left(x_{j}\right)_{*}=y_{j}$ for each $j$, it is clear that the system $\left(K_{R}^{\bullet}(\mathcal{B}), D_{*}^{\bullet}(y)\right)$ is a complex, and coincides with the Aomoto complex of the arrangement $\mathcal{B}$.

We assert that the system $\left(K_{R}^{\bullet}(\mathcal{A}), \Delta_{*}^{\bullet}(y)\right)$ is also a complex, and is chain equivalent to the Aomoto complex of $\mathcal{A}$. For this, consider again the specialization $x \mapsto \mathbf{1}$ corresponding to the trivial local system $\mathcal{L}=\mathbb{C}$. In this instance, as noted above, the boundary maps of $K^{\bullet}(\mathcal{A})$ and $K^{\bullet}(\mathcal{B})$ are all trivial. Thus, for $\mathcal{L}$ trivial, these complexes simply record the cohomology $H^{*}(M ; \mathbb{C})$ and $H^{*}(T ; \mathbb{C})$. As is well known, both algebras are generated in dimension one, and the inclusion $M \subset T$ induces an epimorphism in cohomology. Now recall from the proof of Proposition 2.11 that for $q \leq 1, \Psi^{q}=\mathrm{id} \otimes \psi^{q}$ is constant, and that $\psi^{q}: H^{q}(T ; \mathbb{C}) \rightarrow H^{q}(M ; \mathbb{C})$ is induced by inclusion. So by the naturality of cup products and the continuity of $\Psi^{\bullet}(x)$, we have $\Psi^{q}(\mathbf{1})=\mathrm{id} \otimes \psi^{q}$ for all $q$, where $\psi^{q}: H^{q}(T ; \mathbb{C}) \rightarrow H^{q}(M ; \mathbb{C})$ is induced by $\psi^{1}$.

Identifying the cohomology of $T$ with the exterior algebra, $H^{*}(T ; \mathbb{C})=E$, and the cohomology of $M$ with the Orlik-Solomon algebra, $H^{*}(M ; \mathbb{C})=A(\mathcal{A})$, we realize the map $\Psi^{\bullet}(\mathbf{1})$ as the map on $R$-modules induced by a choice of 
projection $\psi: E \rightarrow A$ from the exterior algebra to the Orlik-Solomon algebra. Consequently, the system of $R$-modules and $R$-linear maps $\left(K_{R}^{\bullet}(\mathcal{A}), \Delta_{*}^{\bullet}(y)\right)$ is a complex, and is chain equivalent to the Aomoto complex of $\mathcal{A}$.

\section{Some consequences}

We discuss some immediate applications of Theorem 2.13.

3.1. Lower bounds. We first show that the Orlik-Solomon algebra cohomology provides a lower bound for the local system cohomology of the complement, recovering a result of Libgober-Yuzvinsky, see [LY, Proposition 4.2, Corollary 4.3]. Fix an arrangement $\mathcal{A}$ of $n$ hyperplanes, with complement $M=M(\mathcal{A})$ and Orlik-Solomon algebra $A=A(\mathcal{A})$. Recall that each weight vector $\lambda \in \mathbb{C}^{n}$ gives rise to an associated rank one local system $\mathcal{L}=\mathcal{L}_{\lambda}$ on $M$.

Proposition 3.2. For each $\lambda \in \mathbb{C}^{n}$ and each $q$, we have

$$
\sup _{m \in \mathbb{Z}^{n}} \operatorname{dim} H^{q}\left(A^{\bullet}, a_{\lambda+m} \wedge\right) \leq \operatorname{dim} H^{q}(M ; \mathcal{L}) .
$$

Proof. First note that this result clearly holds for $\lambda=0$. In general, given $\lambda=\left(\lambda_{1}, \ldots, \lambda_{n}\right) \in \mathbb{C}^{n}$, the cohomology, $H^{*}\left(A^{\bullet}, a_{\lambda} \wedge\right)$, of the Orlik-Solomon algebra is given by that of the specialization, $y \mapsto \lambda$, of the Aomoto complex $\left(A_{R}^{\bullet}(\mathcal{A}), a_{y} \wedge\right)$. Recall that we denote the matrix of $a_{y} \wedge$ by $\mu^{q}(y)$. Similarly, the local system cohomology, $H^{*}(M ; \mathcal{L})$, may be computed from the specialization, $x \mapsto t$, of the universal complex $\left(K_{\Lambda}^{\bullet}(\mathcal{A}), \Delta^{\bullet}(x)\right)$, where $t=\left(t_{1}, \ldots, t_{n}\right) \in\left(\mathbb{C}^{*}\right)^{n}$ satisfies $t_{j}=\exp \left(2 \pi \mathrm{i} \lambda_{j}\right)$, and $\mathcal{L}$ is induced by the representation $\pi_{1}(M) \rightarrow \mathbb{C}^{*}$, $\gamma_{j} \mapsto t_{j}$ for any meridian loop $\gamma_{j}$ about $H_{j} \in \mathcal{A}$.

By Theorem 2.13, the Aomoto complex is chain equivalent to $\left(K_{R}^{\bullet}(\mathcal{A}), \Delta_{*}^{\bullet}(y)\right)$, the linearization of the universal complex. Furthermore, it is well-known that the cohomology, $H^{*}\left(A^{\bullet}, a_{\lambda} \wedge\right)$, of the Orlik-Solomon algebra is invariant under (non-zero) rescaling. Thus for $\lambda$ sufficiently small, the Inverse Function Theorem implies that

$$
\operatorname{rank} \mu^{q}(\lambda)=\operatorname{rank} \Delta_{*}^{q}(\lambda)=\operatorname{rank} \Delta_{*}^{q}(2 \pi \mathrm{i} \lambda)=\operatorname{rank} \Delta^{q}(t)
$$

for each $q$. Thus, $\operatorname{dim} H^{q}\left(A^{\bullet}, a_{\lambda} \wedge\right)=\operatorname{dim} H^{q}(M ; \mathcal{L})$ for $\lambda$ sufficiently small. Hence, using invariance under rescaling for the cohomology of the Orlik-Solomon algebra and upper semicontinuity for the local system cohomology of the complement, we have $\operatorname{dim} H^{q}\left(A^{\bullet}, a_{\lambda} \wedge\right) \leq \operatorname{dim} H^{q}(M ; \mathcal{L})$ for arbitrary $\lambda$.

Now let $m=\left(m_{1}, \ldots, m_{n}\right) \in \mathbb{Z}^{n}$, and recall that the local system associated to $\lambda+m$ coincides with that associated to $\lambda$. Thus, by applying the above considerations to the weight vector $\lambda+m$, we see that $\operatorname{dim} H^{q}\left(A^{\bullet}, a_{\lambda+m} \wedge\right) \leq$ $\operatorname{dim} H^{q}(M ; \mathcal{L})$ for each $q$ and all $m \in \mathbb{Z}^{n}$, which completes the proof.

Remark 3.3. In the case $q=1$, Libgober-Yuzvinsky show that equality holds in (2) for almost all $\lambda$, see [LY, Theorem 5.3]. 
There are local systems $\mathcal{L}$ (resp., weight vectors $\lambda$ ) for which the inequality (2) is strict, as illustrated by the following examples. Both involve central arrangements, where all local system cohomology groups vanish for non-resonant weights. Thus a weight vector which produces cohomology is a priori resonant.

Example 3.4. Let $\mathcal{A}=\left\{H_{1}, \ldots, H_{7}\right\}$ be the central arrangement in $\mathbb{C}^{3}$ with defining polynomial $Q(\mathcal{A})=x(x+y+z)(x+y-z) y(x-y-z)(x-y+z) z$. (The ordering of the hyperplanes corresponds to that of the factors of $Q(\mathcal{A})$.) Consider the weight vector $\lambda=\frac{1}{2}(1,0,0,1,1,0,1) \in \mathbb{C}^{7}$, the associated point $t=(-1,1,1,-1,-1,1,-1) \in\left(\mathbb{C}^{*}\right)^{7}$, and the corresponding local system $\mathcal{L}$ on the complement $M$ of $\mathcal{A}$.

An exercise in the Orlik-Solomon algebra reveals that, for all $m \in \mathbb{Z}^{7}$, we have $\operatorname{dim} H^{1}\left(A^{\bullet}(\mathcal{A}), a_{\lambda+m} \wedge\right) \leq 1$. On the other hand, it is known that the point $t \in \Sigma_{2}^{1}(M)$ is in the second cohomology support locus of (the first cohomology of) $M$, see [CS4, Example 4.4] and Section 3.6 below. It follows that $\operatorname{dim} H^{1}(M(\mathcal{A}), \mathcal{L})=2$, and the inequality (2) is strict for this local system (resp., system of weights).

Example 3.5. A similar example is provided by the Ceva(3) arrangement $\mathcal{A}$ (the monomial arrangement $\left.\mathcal{A}=\mathcal{A}_{3,3,3}\right)$, defined by $Q(\mathcal{A})=\left(x^{3}-y^{3}\right)\left(x^{3}-\right.$ $\left.z^{3}\right)\left(y^{3}-z^{3}\right)$. For the weight vector $\lambda=\frac{1}{3}(1,1,1,1,1,1,-2,-2,-2) \in \mathbb{C}^{9}$ and the corresponding local system $\mathcal{L}$ on the complement $M$ of $\mathcal{A}$, it is known that $\operatorname{dim} H^{1}\left(A^{\bullet}(\mathcal{A}), a_{\lambda+m} \wedge\right) \leq 1$ for all $m \in \mathbb{Z}^{9}$, in particular $\operatorname{dim} H^{1}\left(A^{\bullet}(\mathcal{A}), a_{\lambda} \wedge\right)=$ 1 , and that $\operatorname{dim} H^{1}(M(\mathcal{A}), \mathcal{L})=2$.

3.6. Cohomology support loci and resonance varieties. As another application of Theorem 2.13, we establish the relationship between the cohomology support loci of the complement of an essential arrangement $\mathcal{A}$ in $\mathbb{C}^{\ell}$ and the resonance varieties of its Orlik-Solomon algebra.

Recall that each point $t \in\left(\mathbb{C}^{*}\right)^{n}$ gives rise to a local system $\mathcal{L}=\mathcal{L}_{t}$ on the complement $M=M(\mathcal{A})$. For non-resonant $t$, the cohomology $H^{q}\left(M, \mathcal{L}_{t}\right)$ vanishes for $q<\ell$, see Theorem 1.1. Those $t$ for which $H^{q}\left(M ; \mathcal{L}_{t}\right)$ does not vanish comprise the cohomology support loci

$$
\Sigma_{m}^{q}(M)=\left\{t \in\left(\mathbb{C}^{*}\right)^{n} \mid \operatorname{dim} H^{q}\left(M ; \mathcal{L}_{t}\right) \geq m\right\} .
$$

These loci are algebraic subvarieties of $\left(\mathbb{C}^{*}\right)^{n}$, which are invariants of the homotopy type of $M$. See Arapura [Ar] and Libgober [L1] for detailed discussions of these varieties in the contexts of quasiprojective varieties and plane algebraic curves.

Similarly, each point $\lambda \in \mathbb{C}^{n}$ gives rise to an element $a_{\lambda} \in A^{1}$ of the OrlikSolomon algebra $A=A(\mathcal{A})$. For $q<\ell$, the cohomology $H^{q}\left(A^{\bullet}, a_{\lambda} \wedge\right)$ vanishes for sufficiently generic $\lambda$, see $[\mathrm{Yu}, \mathrm{Fa}]$. Those $\lambda$ for which $H^{q}\left(A^{\bullet}, a_{\lambda} \wedge\right)$ does not vanish comprise the resonance varieties

$$
\mathcal{R}_{m}^{q}(A)=\left\{\lambda \in \mathbb{C}^{n} \mid \operatorname{dim} H^{q}\left(A^{\bullet}, a_{\lambda} \wedge\right) \geq m\right\} .
$$

These subvarieties of $\mathbb{C}^{n}$ are invariants of the Orlik-Solomon algebra $A$. See Falk $[\mathrm{Fa}]$ and Libgober-Yuzvinsky [LY] for detailed discussions of these varieties. 
Recall that $\mathbf{1}=(1, \ldots, 1)$ denotes the identity element of $\left(\mathbb{C}^{*}\right)^{n}$.

Theorem 3.7. Let $\mathcal{A}$ be an arrangement in $\mathbb{C}^{\ell}$ with complement $M$ and OrlikSolomon algebra $A$. Then for each $q$ and $m$, the resonance variety $\mathcal{R}_{m}^{q}(A)$ coincides with the tangent cone of the cohomology support locus $\Sigma_{m}^{q}(M)$ at the point 1.

Proof. For each $t \in\left(\mathbb{C}^{*}\right)^{n}$, the cohomology of $M$ with coefficients in the local system $\mathcal{L}_{t}$ is isomorphic to that of the Morse theoretic complex $\left(K^{\bullet}(\mathcal{A}), \Delta^{\bullet}(t)\right)$, the specialization at $t$ of the universal complex $\left(K_{\Lambda}^{\bullet}(\mathcal{A}), \Delta^{\bullet}(x)\right)$ of Theorem 2.9. So $t \in \Sigma_{m}^{q}(M)$ if and only if $\operatorname{dim} H^{q}\left(K^{\bullet}(\mathcal{A}), \Delta^{\bullet}(t)\right) \geq m$. An exercise in linear algebra shows that

$$
\Sigma_{m}^{q}(M)=\left\{t \in\left(\mathbb{C}^{*}\right)^{n} \mid \operatorname{rank} \Delta^{q-1}(t)+\operatorname{rank} \Delta^{q}(t) \leq \operatorname{dim} K^{q}(\mathcal{A})-m\right\} .
$$

Results of Arapura [Ar] show that $\Sigma_{m}^{q}(M)$ may be realized as the transverse intersection of smooth subvarieties of $\left(\mathbb{C}^{*}\right)^{n}$. So the tangent cone at $\mathbf{1}$ is given by

$$
\mathrm{C}_{\mathbf{1}} \Sigma_{m}^{q}(M)=\left\{\lambda \in \mathbb{C}^{n} \mid \operatorname{rank} \Delta_{*}^{q-1}(\lambda)+\operatorname{rank} \Delta_{*}^{q}(\lambda) \leq \operatorname{dim} K^{q}(\mathcal{A})-m\right\} .
$$

For $\lambda \in \mathbb{C}^{n}$, we have $\lambda \in \mathcal{R}_{m}^{q}$ if $\operatorname{dim} H^{q}\left(A^{\bullet}, a_{\lambda} \wedge\right) \geq m$. Denote the matrix of $a_{\lambda} \wedge: A^{q}(\mathcal{A}) \rightarrow A^{q+1}(\mathcal{A})$ by $\mu^{q}(\lambda)$. Then, as above,

$$
\mathcal{R}_{m}^{q}(A)=\left\{\lambda \in \mathbb{C}^{n} \mid \operatorname{rank} \mu^{q-1}(\lambda)+\operatorname{rank} \mu^{q}(\lambda) \leq \operatorname{dim} A^{q}(\mathcal{A})-m\right\} .
$$

Now $\operatorname{dim} A^{q}(\mathcal{A})=\operatorname{dim} K^{q}(\mathcal{A})=b_{q}(\mathcal{A})$ and for each $q$, by Theorem 2.13, we have $\operatorname{rank} \mu^{q}(\lambda)=\operatorname{rank} \Delta_{*}^{q}(\lambda)$. Thus,

$$
\mathcal{R}_{m}^{q}(A)=\left\{\lambda \in \mathbb{C}^{n} \mid \operatorname{rank} \Delta_{*}^{q-1}(\lambda)+\operatorname{rank} \Delta_{*}^{q}(\lambda) \leq b_{q}(\mathcal{A})-m\right\},
$$

and the result follows.

The cohomology support loci are known to be unions of torsion-translated subtori of $\left(\mathbb{C}^{*}\right)^{n}$, see [Ar, Corollary V.1.2]. In particular, all irreducible components of $\Sigma_{m}^{q}(M)$ passing through 1 are subtori of $\left(\mathbb{C}^{*}\right)^{n}$. Consequently, all irreducible components of the tangent cone are linear subspaces of $\mathbb{C}^{n}$. So we have the following.

Corollary 3.8. For each $q$ and $m$, the resonance variety $\mathcal{R}_{m}^{q}(A)$ is the union of an arrangement of subspaces in $\mathbb{C}^{n}$.

Remark 3.9. Several special cases of Theorem 3.7 and Corollary 3.8 were previously known. For $q=1$, these results were established by Cohen-Suciu [CS4], see also Libgober-Yuzvinsky [L1, LY]. For the discriminantal arrangements of Schechtman-Varchenko [SV], they were established in [C3]. In particular, as conjectured by Falk [Fa, Conjecture 4.7], the resonance varieties $\mathcal{R}_{m}^{q}(A)$ were known to be unions of linear subspaces in these instances. Corollary 3.8 above resolves this conjecture positively for all arrangements in all dimensions.

Remark 3.10. Theorem 3.7 and Corollary 3.8 have been obtained recently by Libgober in a more general situation, see [L2]. 


\section{Rational local systems}

Let $\mathcal{A}=\left\{H_{1}, \ldots, H_{n}\right\}$ be an arrangement of complex hyperplanes, and let $\lambda=\left(\lambda_{1}, \ldots, \lambda_{n}\right)$ be a system of rational weights, $\lambda_{j}=k_{j} / N$, where $k_{j} \in \mathbb{Z}$ and $N \in \mathbb{N}$. The representation $\rho: \pi_{1}(M) \rightarrow \mathbb{C}^{*}, \gamma_{j} \mapsto t_{j}=\exp \left(2 \pi \mathrm{i} \lambda_{j}\right)$, is unitary, and we call the associated local system $\mathcal{L}$ on $M$ rational. Since our primary interest is in this local system, we may assume without loss that the greatest common divisor of the integers $k_{j}$ is relatively prime to $N$. In this section, we obtain combinatorial upper bounds on the local system Betti numbers $\operatorname{dim}_{\mathbb{C}} H^{q}(M ; \mathcal{L})$. We then use these bounds to study non-resonant local systems.

4.1. Combinatorial cohomology $\bmod N$. Let $A_{\mathbb{Q}}(\mathcal{A})$ be the Orlik-Solomon algebra of $\mathcal{A}$, with rational coefficients, and generated by $\left\{a_{1}, \ldots, a_{n}\right\}$. If the underlying arrangement is clear, we write $A_{\mathbb{Q}}=A_{\mathbb{Q}}(\mathcal{A})$. Left-multiplication by the element $a_{\lambda}=\sum \lambda_{j} a_{j} \in A_{\mathbb{Q}}^{1}$ induces a differential on the Orlik-Solomon algebra, and we denote the resulting complex by $\left(A_{\mathbb{Q}}^{\bullet}, a_{\lambda} \wedge\right)$. Similarly, associated to the element $a_{k}=N a_{\lambda}=\sum k_{j} a_{j}$, we have the complex $\left(A_{\mathbb{Q}}^{\bullet}, a_{k} \wedge\right)$.

Lemma 4.2. The complexes $\left(A_{\mathbb{Q}}^{\bullet}, a_{\lambda} \wedge\right)$ and $\left(A_{\mathbb{Q}}^{\bullet}, a_{k} \wedge\right)$ are chain equivalent.

Proof. Define $\eta: A_{\mathbb{Q}}^{\bullet} \rightarrow A_{\mathbb{Q}}^{\bullet}$ by $\eta(a)=N^{q} a$ for $a \in A_{\mathbb{Q}}^{q}$. Since $\eta$ is clearly an isomorphism, it is enough to show that $\eta$ is a chain map. For $a$ as above, we have

$$
\eta\left(a_{\lambda} \wedge a\right)=N^{q+1} \sum \frac{k_{j}}{N} a_{j} \wedge a=N^{q} a_{k} \wedge a=a_{k} \wedge \eta(a)
$$

and $\eta$ is a chain map.

Denote the matrix of $a_{\lambda} \wedge: A_{\mathbb{Q}}^{q} \rightarrow A_{\mathbb{Q}}^{q+1}$ by $\mu^{q}(\lambda)$, and that of $a_{k} \wedge: A_{\mathbb{Q}}^{q} \rightarrow$ $A_{\mathbb{Q}}^{q+1}$ by $\mu^{q}(k)$. The entries of the latter are integers, so we consider the OrlikSolomon algebra with integer coefficients, the associated complex $\left(A_{\mathbb{Z}}^{\bullet}, a_{k} \wedge\right)$, and the reduction of this complex $\bmod N$. Let $\left(A_{N}^{\bullet}, \bar{a}_{k} \wedge\right)$ be the reduction of $\left(A_{\mathbb{Z}}^{\bullet}, a_{k} \wedge\right) \bmod N$, where $A_{N}=A_{\mathbb{Z}_{N}}$ denotes the Orlik-Solomon algebra with coefficients in the ring $\mathbb{Z}_{N}$ and $\bar{a}_{k}=a_{k} \bmod N$. Denote the matrix of $\bar{a}_{k} \wedge$ : $A_{N}^{q} \rightarrow A_{N}^{q+1}$ by $\bar{\mu}^{q}(k)$.

4.3. Upper bounds. We now obtain combinatorial upper bounds on the Betti numbers, $\operatorname{dim}_{\mathbb{C}} H^{q}(M ; \mathcal{L})$, for a rational local system $\mathcal{L}$. We shall make use of the following elementary fact.

Lemma 4.4. Let $\zeta=\exp (2 \pi \mathrm{i} / N)$ be a primitive $N$-th root of unity, and let $f(z) \in \mathbb{C}\left[z^{ \pm 1}\right]$ be a Laurent polynomial which satisfies $f(1)=0, f(\zeta)=0$, and $f^{\prime}(1) \in \mathbb{Z}$. Then $\left[f^{\prime}(1)\right]$ is a zero-divisor in $\mathbb{Z}_{N}$.

Proof. Write $f(z)=z^{-m} p(z)$, where $p(z) \in \mathbb{C}[z]$ is a polynomial. Since $p(\zeta)=$ $f(\zeta)=0$, we have $p(z)=\Phi_{N}(z) \cdot q(z)$, where $\Phi_{N}(z)$ is the $N$-th cyclotomic polynomial and $q(z) \in \mathbb{C}[z]$. Since $p(1)=f(1)=0$ and $\Phi_{N}(1) \neq 0$, we also have $q(1)=0$. These considerations, and a brief calculation, reveal that $f^{\prime}(1)=$ 
$p^{\prime}(1)=\Phi_{N}(1) \cdot q^{\prime}(1)$. Since $\Phi_{N}(1)$ divides $N$, we see that $\left[f^{\prime}(1)\right]$ is a zero-divisor in $\mathbb{Z}_{N}$.

The main result of this section is the following.

Theorem 4.5. Let $\lambda=k / N$ be a system of rational weights, and let $\mathcal{L}$ be the associated rational local system on the complement $M$ of $\mathcal{A}$. Then, for each $q$,

$$
\operatorname{dim}_{\mathbb{C}} H^{q}(M ; \mathcal{L}) \leq \operatorname{rank}_{\mathbb{Z}_{N}} H^{q}\left(A_{N}^{\bullet}, \bar{a}_{k} \wedge\right) .
$$

Proof. For any system of rational weights $\lambda=\frac{1}{N}\left(k_{1}, \ldots, k_{n}\right)$, the rational local system $\mathcal{L}$ is determined by the unitary representation $\rho: \pi_{1}(M) \rightarrow \mathbb{C}^{*}$ defined by $\gamma_{j} \mapsto \zeta^{k_{j}}$, where $\zeta=\exp (2 \pi \mathrm{i} / N)$. This representation factors through the integers $\mathbb{Z}=\langle z\rangle$ as follows. Define $\xi: \pi_{1}(M) \rightarrow \mathbb{Z}$ by $\xi\left(\gamma_{j}\right)=z^{k_{j}}$ and define $\chi: \mathbb{Z} \rightarrow \mathbb{C}^{*}$ by $\chi(z)=\zeta$. We then have $\rho=\chi \circ \xi$.

The cohomology of $M$ with coefficients in $\mathcal{L}$ is isomorphic to that of the complex $\left(K^{\bullet}(\mathcal{A}), \Delta^{\bullet}(t)\right)$, the specialization of the universal complex $\left(K_{\Lambda}^{\bullet}(\mathcal{A}), \Delta^{\bullet}(x)\right)$ at the point $t=\left(t_{1}, \ldots, t_{n}\right) \in\left(\mathbb{C}^{*}\right)^{n}$, where $t_{j}=\exp \left(2 \pi \mathrm{i} k_{j} / N\right)=\zeta^{k_{j}}$. Thus for a rational local system, via the map $\Lambda \rightarrow \mathbb{C}\left[z^{ \pm 1}\right]$ defined by $t_{j} \mapsto z^{k_{j}}$, the specialization map $\Lambda \rightarrow \mathbb{C}$ factors through the ring of Laurent polynomials in the single variable $z$. To emphasize the dependence of the boundary maps of $K^{\bullet}(\mathcal{A})$ on $\zeta$, we shall write $\Delta^{\bullet}(t)=\Delta^{\bullet}(\zeta)$. By virtue of the above factorization, for each $q$, the matrix $\Delta^{q}(\zeta)$ may be realized as the specialization, $z \mapsto \zeta$, of a matrix $\Delta^{q}(z)$ with entries in $\mathbb{C}\left[z^{ \pm 1}\right]$.

By Theorem 2.13, the Aomoto complex $\left(A_{R}^{\bullet}(\mathcal{A}), a_{y} \wedge\right)$ is chain equivalent to the linearization, $\left(K_{R}^{\bullet}(\mathcal{A}), \Delta_{*}^{\bullet}(y)\right)$, of the universal complex $\left(K_{\Lambda}^{\bullet}(\mathcal{A}), \Delta^{\bullet}(x)\right)$. Both this chain equivalence and the construction of the Aomoto complex involve choosing a basis for the Orlik-Solomon algebra of $\mathcal{A}$. Making these choices in a consistent manner, we may assume without loss that the two complexes coincide, $\left(K_{R}^{\bullet}(\mathcal{A}), \Delta_{*}^{\bullet}(y)\right)=\left(A_{R}^{\bullet}(\mathcal{A}), a_{y} \wedge\right)$. Specializing, $y \mapsto k$, yields the complex $\left(A_{\mathbb{C}}, a_{k} \wedge\right)=\left(K^{\bullet}(\mathcal{A}), \Delta_{*}^{\bullet}(k)\right)$. Recall that we denote the matrix of $a_{k} \wedge$ : $A^{q} \rightarrow A^{q+1}$ by $\mu^{q}(k)$. Since for each $j$, we have $t_{j}=\chi \circ \xi\left(x_{j}\right)=\chi\left(z^{k_{j}}\right)=\zeta^{k_{j}}$, these boundary maps may be realized as the derivatives at $z=1$ of the maps $\Delta^{q}(z)$ noted above: $\mu^{q}(k)=\Delta_{*}^{q}(k)=\left.\frac{d}{d z} \Delta^{q}(z)\right|_{z=1}$.

Recall from Lemma 2.12 that the boundary maps of the Aomoto complex consist of integral linear combinations of the indeterminates $y_{j}$. Thus the entries of the matrices $\mu^{q}(k)=\Delta_{*}^{q}(k)$ above are integers, and, as in Section 4.1 above, we may consider the complex $\left(A_{\mathbb{Z}}^{\bullet}, a_{k} \wedge\right)$. The reduction, $\left(A_{N}^{\bullet}, \bar{a}_{k} \wedge\right)$, of this complex $\bmod N$ has boundary maps given by the matrices $\bar{\mu}^{q}(k)$, the reductions $\bmod N$ of $\mu^{q}(k)=\Delta_{*}^{q}(k)$. By Lemma 4.4, if a minor of $\bar{\mu}^{q}(k)$ is a unit in $\mathbb{Z}_{N}$, then the corresponding minor of $\Delta^{q}(\zeta)$ is non-zero. Thus, $\operatorname{rank}_{\mathbb{C}} \Delta^{q}(\zeta) \geq \operatorname{rank}_{\mathbb{Z}_{N}} \bar{\mu}^{q}(k)$, and the result follows.

There are rational local systems $\mathcal{L}$ (resp., weight vectors $\lambda$ ) for which the inequality (3) is strict, as illustrated by the following example. 
Example 4.6. Let $\mathcal{A}=\left\{H_{1}, \ldots, H_{8}\right\}$ be a realization of the MacLane $\left(8_{3}\right)$ configuration, with defining polynomial

$$
Q(\mathcal{A})=x y(y-x) z\left(z-x-\omega^{2} y\right)(z+\omega y)(z-x)\left(z+\omega^{2} x+\omega y\right)
$$

where $\omega$ is a primitive third root of unity and the ordering of the hyperplanes of $\mathcal{A}$ corresponds to that of the factors of $Q(\mathcal{A})$. Consider the rational weights

$$
\lambda(u, v)=\frac{u}{3}(1,0,2,1,2,2,1,0)+\frac{v}{3}(2,2,2,1,1,0,0,1),
$$

where $u, v \in\{0,1,2\}$, the associated points $t(u, v)=\exp (2 \pi \mathrm{i} \lambda(u, v))$ in the complex torus $\left(\mathbb{C}^{*}\right)^{8}$, and the corresponding rational local systems $\mathcal{L}=\mathcal{L}(u, v)$ on the complement $M$ of $\mathcal{A}$.

As observed by Matei-Suciu [MS, Example 5.9], the mod 3 resonance variety, $\mathcal{R}_{1}^{1}\left(A, \mathbb{Z}_{3}\right)$, of the Orlik-Solomon algebra of $\mathcal{A}$ contains a two-dimensional component. In our notation, this component may be described by the equations

$$
\left\{\begin{array}{l|c}
\bar{k}=\left(k_{1}, \ldots, k_{8}\right) \in\left(\mathbb{Z}_{3}\right)^{8} & \begin{array}{c}
k_{1}+k_{5}=k_{2}+k_{8}=k_{3}+k_{4}=k_{6}+k_{7}=0 \\
k_{3}+k_{5}+k_{6}=k_{3}+k_{7}+k_{8}=0
\end{array}
\end{array}\right\} .
$$

The (nine) points of $C$ may be realized as the reductions mod 3 of the integral weight vectors $k(u, v)=3 \lambda(u, v)$ for $u, v \in\{0,1,2\}$. Thus for $k=k(u, v)$ with $(u, v) \neq(0,0)$, we have $\operatorname{rank}_{\mathbb{Z}_{3}} H^{1}\left(A_{3}, \bar{a}_{k} \wedge\right)=1$, as may be readily checked by direct calculation in the Orlik-Solomon algebra.

On the other hand, for each of the non-trivial rational local systems $\mathcal{L}=$ $\mathcal{L}(u, v)$, we have $H^{1}(M ; \mathcal{L})=0$. Using the braided wiring diagram for this arrangement recorded in [CS3, Example 8.6], one may compute the braid monodromy of $\mathcal{A}$, and the ensuing braid monodromy presentation of the fundamental group of $M$, see [CS2]. Then an exercise with this presentation and the Fox calculus reveals that the first local system cohomology of $M$ is trivial, $H^{1}(M ; \mathcal{L})=0$, for each of the local systems $\mathcal{L}=\mathcal{L}(u, v)$. Thus, for each of the weight vectors $\lambda(u, v)$, the inequality (3) is strict.

Note that, since $H^{1}(M ; \mathcal{L})=0$ for $\mathcal{L}=\mathcal{L}(u, v)$, we have $H^{1}\left(A_{\mathbb{C}}^{\bullet} a_{\lambda+m} \wedge\right)=0$ for every integral translate $m \in \mathbb{Z}^{8}$ of each of the weight vectors $\lambda=\lambda(u, v)$.

Example 4.7. Using the previous examples, one can construct arrangements and weight vectors for which both inequalities (2) and (3) are simultaneously strict.

Let $\mathcal{A}^{\prime}$ be a generic section in $\mathbb{C}^{2}$ of the Ceva(3) arrangement from Example 3.5, and let $\mathcal{A}^{\prime \prime}$ be a generic section in $\mathbb{C}^{2}$ of the Maclane arrangement above. Consider the weight vectors $\lambda^{\prime}=\frac{1}{3} k^{\prime}$ for $\mathcal{A}^{\prime}$ and $\lambda^{\prime \prime}=\frac{1}{3} k^{\prime \prime}$ for $\mathcal{A}^{\prime \prime}$, where

$k^{\prime}=(1,1,1,1,1,1,-2,-2,-2) \in \mathbb{Z}^{9} \quad$ and $\quad k^{\prime \prime}=(1,0,-1,1,-1,-1,1,0) \in \mathbb{Z}^{8}$.

Note that $\lambda^{\prime \prime}$ is an integral translate of the weight vector $\lambda(1,0)$ from the previous example and that $k^{\prime \prime} \equiv k(1,0) \bmod 3$. Let $\mathcal{L}^{\prime}$ and $\mathcal{L}^{\prime \prime}$ denote the local systems on $M\left(\mathcal{A}^{\prime}\right)$ and $M\left(\mathcal{A}^{\prime \prime}\right)$ corresponding to $\lambda^{\prime}$ and $\lambda^{\prime \prime}$. Since these arrangements are generic sections, our previous calculations and Euler characteristic arguments yield the Orlik-Solomon algebra cohomology and local system cohomology. 
We record this information using Poincaré polynomials. Write

$$
\begin{aligned}
\left.P\left(A_{\mathbb{C}}^{\bullet} \mathcal{A}\right), \lambda, t\right) & =\sum \operatorname{dim}_{\mathbb{C}} H^{i}\left(A_{\mathbb{C}}^{\bullet}(\mathcal{A}), a_{\lambda} \wedge\right) t^{i}, \\
P(M(\mathcal{A}), \mathcal{L}, t) & =\sum \operatorname{dim}_{\mathbb{C}} H^{i}(M(\mathcal{A}), \mathcal{L}) t^{i}, \text { and } \\
P\left(A_{N}^{\bullet}(\mathcal{A}), k, t\right) & =\sum \operatorname{rank}_{\mathbb{Z}_{N}} H^{i}\left(A_{N}^{\bullet}(\mathcal{A}), \bar{a}_{k} \wedge\right) t^{i}
\end{aligned}
$$

Then, for $\mathcal{A}^{\prime}$, we have

$$
P\left(A_{\mathbb{C}}^{\bullet}\left(\mathcal{A}^{\prime}\right), \lambda^{\prime}, t\right)=t+17 t^{2} \quad \text { and } \quad P\left(M\left(\mathcal{A}^{\prime}\right), \mathcal{L}^{\prime}, t\right)=2 t+18 t^{2} .
$$

Furthermore, a computation in the Orlik-Solomon algebra reveals that

$$
P\left(A_{3}^{\bullet}\left(\mathcal{A}^{\prime}\right), k^{\prime}, t\right)=P\left(M\left(\mathcal{A}^{\prime}\right), \mathcal{L}^{\prime}, t\right) .
$$

For $\mathcal{A}^{\prime \prime}$, we have

$P\left(A_{\mathbb{C}}^{\bullet}\left(\mathcal{A}^{\prime \prime}\right), \lambda^{\prime \prime}, t\right)=P\left(M\left(\mathcal{A}^{\prime \prime}\right), \mathcal{L}^{\prime \prime}, t\right)=13 t^{2} \quad$ and $\quad P\left(A_{3}^{\bullet}\left(\mathcal{A}^{\prime \prime}\right), k^{\prime \prime}, t\right)=t+14 t^{2}$.

Now let $\mathcal{A}=\mathcal{A}^{\prime} \times \mathcal{A}^{\prime \prime}$ be the product arrangement in $\mathbb{C}^{4}$, and let $k=\left(k^{\prime}, k^{\prime \prime}\right) \in$ $\mathbb{Z}^{17}$. Consider the weight vector $\lambda=\frac{1}{3} k=\left(\lambda^{\prime}, \lambda^{\prime \prime}\right)=\frac{1}{3}\left(k^{\prime}, k^{\prime \prime}\right) \in \mathbb{C}^{17}$, and the associated local system $\mathcal{L}$ on $M(\mathcal{A})$. By construction, $\mathcal{L}$ is the product local system on $M(\mathcal{A}) \cong M\left(\mathcal{A}^{\prime}\right) \times M\left(\mathcal{A}^{\prime \prime}\right)$. Similarly, the boundary map of the Orlik-Solomon algebra complex is compatible with the product structure $A(\mathcal{A}) \simeq A\left(\mathcal{A}^{\prime}\right) \times A\left(\mathcal{A}^{\prime \prime}\right)$. Consequently, we may use the Künneth formula to obtain

$$
\begin{aligned}
& P\left(A_{\mathbb{C}}^{\bullet}(\mathcal{A}), \lambda, t\right)=P\left(A_{\mathbb{C}}^{\bullet}\left(\mathcal{A}^{\prime}\right), \lambda^{\prime}, t\right) \cdot P\left(A_{\mathbb{C}}^{\bullet}\left(\mathcal{A}^{\prime \prime}\right), \lambda^{\prime \prime}, t\right)=13 t^{3}+221 t^{4}, \\
& P(M(\mathcal{A}), \mathcal{L}, t)=P\left(M\left(\mathcal{A}^{\prime}\right), \mathcal{L}^{\prime}, t\right) \cdot P\left(M\left(\mathcal{A}^{\prime \prime}\right), \mathcal{L}^{\prime \prime}, t\right)=26 t^{3}+234 t^{4}, \text { and } \\
& P\left(A_{3}^{\bullet}(\mathcal{A}), k, t\right)=P\left(A_{3}^{\bullet}\left(\mathcal{A}^{\prime}\right), k^{\prime}, t\right) \cdot P\left(A_{3}^{\bullet}\left(\mathcal{A}^{\prime \prime}\right), k^{\prime \prime}, t\right)=2 t^{2}+46 t^{3}+252 t^{4} .
\end{aligned}
$$

Thus,

$$
\operatorname{dim}_{\mathbb{C}} H^{i}\left(A_{\mathbb{C}}^{\bullet}(\mathcal{A}), a_{\lambda} \wedge\right)<\operatorname{dim}_{\mathbb{C}} H^{i}(M(\mathcal{A}), \mathcal{L})<\operatorname{rank}_{\mathbb{Z}_{3}} H^{i}\left(A_{3}^{\bullet}(\mathcal{A}), \bar{a}_{k} \wedge\right)
$$

for $i=3,4$, and both inequalities (2) and (3) are strict.

4.8. Resonance. We use the results obtained above to study resonance phenomena. Let $\mathcal{A}$ be an essential arrangement of $n$ hyperplanes in $\mathbb{C}^{\ell}$, with complement $M$. We call a system of weights $\lambda \in \mathbb{C}^{n}$ non-resonant if the Betti numbers of $M$ with coefficients in the associated local system $\mathcal{L}$ are minimal. Since the boundary maps $\Delta^{q}(t)$ of the complex $K^{\bullet}(\mathcal{A})$ computing the local system cohomology generically take on their maximal ranks, the set of non-resonant weights is open and dense in $\mathbb{C}^{n}$. We denote this set by $\mathbf{U}=\mathbf{U}(\mathcal{A})$. It may be described as the set of all $\lambda \in \mathbb{C}^{n}$ for which the sum, $\Sigma(\mathcal{A})=\sum_{q=0}^{\ell} \operatorname{dim} H^{q}(M ; \mathcal{L})$, of the local system Betti numbers is minimal.

Recall that an edge is a nonempty intersection of hyperplanes. An edge is called dense if the subarrangement of hyperplanes containing it is irreducible: the hyperplanes cannot be partitioned into nonempty sets so that after a change of coordinates hyperplanes in different sets are in different coordinates. This is a combinatorially determined condition, see [STV]. For each edge $X$, define 
$\lambda_{X}=\sum_{X \subseteq H_{j}} \lambda_{j}$. Let $\mathcal{A}_{\infty}=\mathcal{A} \cup H_{\infty}$ denote the projective closure of $\mathcal{A}$, the union of $\mathcal{A}$ and the hyperplane at infinity in $\mathbb{C P}^{\ell}$, see [OT2]. Consider the following sets:

$$
\begin{aligned}
\mathbf{V}=\mathbf{V}(\mathcal{A}) & =\left\{\lambda \in \mathbb{C}^{n} \mid \lambda_{X} \notin \mathbb{Z}_{>0} \text { for every dense edge } X \text { of } \mathcal{A}_{\infty}\right\}, \\
\mathbf{W}=\mathbf{W}(\mathcal{A}) & =\left\{\lambda \in \mathbb{C}^{n} \mid \lambda_{X} \notin \mathbb{Z}_{\geq 0} \text { for every dense edge } X \text { of } \mathcal{A}_{\infty}\right\} .
\end{aligned}
$$

Theorem 4.9. If $\lambda \in \mathbf{W}$, then $H^{q}(M ; \mathcal{L})=0$ for $q \neq \ell$ and $\operatorname{dim} H^{\ell}(M ; \mathcal{L})=$ $|e(M)|$.

Since $\operatorname{dim} K^{q}(\mathcal{A})=b_{q}(\mathcal{A})=\operatorname{dim}_{\mathbb{C}} H^{q}(M ; \mathbb{C})$, we see that this theorem minimizes $\Sigma(\mathcal{A})$. It follows that $\mathbf{W} \subset \mathbf{U}$, proving Theorem 1.1 of the Introduction. There is an important difference between these statements, however. Theorem 1.1 is an existence result which does not reveal the structure of $\mathbf{U}$, while Theorem 4.9 identifies a large subset of $\mathbf{U}$. Here we state the two ingredients of the proof of Theorem 4.9 more precisely. Choose a degree one polynomial $\alpha_{j}$ with kernel $H_{j} \in \mathcal{A}$ for $1 \leq j \leq n$. The Brieskorn algebra, $B(\mathcal{A})$, is the graded $\mathbb{C}$-algebra generated by 1 and the holomorphic 1 -forms $\omega_{j}=d \alpha_{j} / \alpha_{j}$. Let $\omega_{\lambda}=\sum_{j=1}^{n} \lambda_{j} \omega_{j}$. Then $\left(B^{\bullet}(\mathcal{A}), \omega_{\lambda} \wedge\right)$ is a subcomplex of the twisted de Rham complex used to calculate $H^{*}(M ; \mathcal{L})$. The results establishing Theorem 4.9 are:

1. If $\lambda \in \mathbf{V}$, then $H^{*}(M ; \mathcal{L}) \simeq H^{*}\left(B^{\bullet}(\mathcal{A}), \omega_{\lambda} \wedge\right)$. This is a result of EsnaultSchechtman-Viehweg [ESV], obtained by using Deligne's work [De], and refined by Schechtman-Terao-Varchenko [STV]. The condition $\lambda \in \mathbf{V}$ is a monodromy condition, imposed on a normal crossing divisor obtained by resolution of singularities.

2. The cohomology, $H^{*}\left(B^{\bullet}(\mathcal{A}), \omega_{\lambda} \wedge\right)$, of the Brieskorn algebra is isomorphic to that of the Orlik-Solomon algebra, $H^{*}\left(A^{\bullet}(\mathcal{A}), a_{\lambda} \wedge\right)$, [OT2]. The latter was studied by Yuzvinsky [Yu], who showed that if $\lambda \in \mathbf{W}$, then $H^{q}\left(A^{\bullet}(\mathcal{A}), a_{\lambda} \wedge\right)=0$ for $q \neq \ell$, from which it follows that

$$
\operatorname{dim} H^{\ell}\left(A^{\bullet}(\mathcal{A}), a_{\lambda} \wedge\right)=|e(M)| \text {. }
$$

We give a proof of Theorem 1.1 which uses only Theorem 4.5 and Yuzvinsky's result stated above, and thus avoids use of resolution of singularities.

Proof of Theorem 1.1. The alternating sum of the dimensions of the cochain groups $K^{q}(\mathcal{A})$ is the Euler characteristic, so it suffices to prove that there exists a system of weights with cohomology groups as stated in the theorem. Yuzvinsky's argument may be applied with weights in an arbitrary field, and requires only that $\lambda_{X}$ be an invertible element for all dense edges.

Choose a prime $p>n$ and let $\lambda=k / p$, where $k_{j}=1$ for every $j$. Then for every (dense) edge $X, k_{X}=|X| \leq n<p$. Thus $k_{X}$ is a unit in $\mathbb{Z}_{p}$, and $H^{q}\left(A_{p}^{\bullet}(\mathcal{A}), \bar{a}_{k} \wedge\right)=0$ for $q \neq \ell$. So by Theorem 4.5 , for the rational local system $\mathcal{L}$ corresponding to $\lambda$, we have $H^{q}(M, \mathcal{L})=0$ for $q \neq \ell$, and we are done.

Remark 4.10. There are in general many non-resonant weights which do not lie in the set $\mathbf{W}$. For instance, we have $\lambda(1,0) \in \mathbf{U} \backslash \mathbf{W}$ in Example 4.6. Turning 
to resonant weights, the arrangement of three lines through the origin in $\mathbb{C}^{2}$ with weights $\lambda=\left(\lambda_{1}, \lambda_{2}, \lambda_{3}\right) \in \mathbf{V}$ satisfying $\lambda_{1}+\lambda_{2}+\lambda_{3}=0$ shows that $\mathbf{V} \nsubseteq \mathbb{U}$. Examples 3.4 and 3.5 show that there are resonant weights $\lambda$ for which $\lambda+m \notin \mathbf{V}$ for any $m \in \mathbb{Z}^{n}$. The following summarizes our current understanding of resonant weights:

1. If $\lambda+m \in \mathbf{V}$ for some $m \in \mathbb{Z}^{n}$, then $H^{*}(M ; \mathcal{L}) \simeq H^{*}\left(A_{\mathbb{C}}^{\bullet}, a_{\lambda+m} \wedge\right)$ and we have an effective algorithm.

2. Otherwise, we have only the inequalities

$$
\operatorname{dim}_{\mathbb{C}} H^{q}\left(A_{\mathbb{C}}^{\bullet}, a_{\lambda} \wedge\right) \leq \operatorname{dim}_{\mathbb{C}} H^{q}(M ; \mathcal{L}) \leq \operatorname{dim}_{\mathbb{C}} H^{q}(M ; \mathbb{C})
$$

for arbitrary weights, and

$$
\operatorname{dim}_{\mathbb{C}} H^{q}\left(A_{\mathbb{C}}^{\bullet}, a_{\lambda} \wedge\right) \leq \operatorname{dim}_{\mathbb{C}} H^{q}(M ; \mathcal{L}) \leq \operatorname{rank}_{\mathbb{Z}_{N}} H^{q}\left(A_{N}^{\bullet}, \bar{a}_{k} \wedge\right)
$$

for rational weights.

Acknowledgment. Portions of this work were carried out in the Spring of 1999, when the first author served as an Honorary Fellow in the Department of Mathematics at the University of Wisconsin-Madison. He thanks the Department for its hospitality, and for providing an exciting and productive mathematical environment. He also thanks the College of Arts \& Sciences at Louisiana State University for granting the Research Fellowship which made this visit possible.

\section{References}

[Ao] K. Aomoto, Un théorème du type de Matsushima-Murakami concernant l'intégrale des fonctions multiformes, J. Math. Pures Appl. 52 (1973), 1-11.

[AK] K. Aomoto and M. Kita, Hypergeometric Functions (in Japanese), Springer-Verlag, Tokyo, 1994.

[Ar] D. Arapura, Geometry of cohomology support loci for local systems I, J. Alg. Geom. 6 (1997), 563-597.

[C1] D. Cohen, Cohomology and intersection cohomology of complex hyperplane arrangements, Adv. Math. 97 (1993), 231-266.

[C2] Morse inequalities for arrangements, Adv. Math. 134 (1998), 43-45.

[C3] - On the cohomology of discriminantal arrangements and Orlik-Solomon algebras, in: Arrangements - Tokyo 1998, Adv. Stud. Pure Math., Math. Soc. Japan, Tokyo, to appear.

[CS1] D. Cohen and A. Suciu, On Milnor fibrations of arrangements, J. London Math. Soc. 51 (1995), 105-119.

[CS2] - The braid monodromy of plane algebraic curves and hyperplane arrangements, Comment. Math. Helv. 72 (1997), 285-315.

[CS3] - Alexander invariants of complex hyperplane arrangements, Trans. Amer. Math. Soc. 351 (1999), 4043-4067.

[CS4] - Characteristic varieties of arrangements, Math. Proc. Cambridge Philos. Soc. 127 (1999), 33-54.

[De] P. Deligne, Equations Différentielles à Points Singuliers Réguliers, Lect. Notes in Math., vol. 163, Springer-Verlag, Berlin-New York, 1970.

[ESV] H. Esnault, V. Schechtman, and V. Viehweg, Cohomology of local systems on the complement of hyperplanes, Invent. Math. 109 (1992), 557-561. Erratum, ibid. 112 (1993), 447.

[Fa] M. Falk, Arrangements and cohomology, Ann. Comb. 1 (1997), 135-157. 
[FT] M. Falk and H. Terao, Bnbc-bases for cohomology of local systems on hyperplane complements, Trans. Amer. Math. Soc. 349 (1997), 189-202.

[Ge] I.M. Gelfand, General theory of hypergeometric functions, Soviet Math. Dokl. 33 (1986), 573-577.

[GM] M. Goresky and R. MacPherson, Stratified Morse Theory, Ergeb. Math. Grenzgeb., vol. 14, Springer-Verlag, Berlin-New York, 1988.

[Ha] A. Hattori, Topology of $\mathbb{C}^{n}$ minus a finite number of affine hyperplanes in general position, J. Fac. Sci. Univ. Tokyo 22 (1975), 205-219.

[Ko] T. Kohno, Homology of a local system on the complement of hyperplanes, Proc. Japan Acad. Ser. A 62 (1986), 144-147.

[L1] A. Libgober, Characteristic varieties of algebraic curves, preprint, 1998.

[L2] - First order deformations for rank one local systems with non vanishing cohomology, preprint, 1999.

[LY] A. Libgober, and S. Yuzvinsky, Cohomology of the Orlik-Solomon algebras and local systems, Compositio Math., to appear.

[Ma] D. Massey, Numerical invariants of perverse sheaves, Duke Math. J. 73 (1994), 307369.

[MS] D. Matei and A. Suciu, Cohomology rings and nilpotent quotients of real and complex arrangements, in: Arrangements - Tokyo 1998, Adv. Stud. Pure Math., Math. Soc. Japan, Tokyo, to appear.

[OT1] P. Orlik and H. Terao, Arrangements of Hyperplanes, Grundlehren Math. Wiss., vol. 300, Springer-Verlag, Berlin, 1992.

[OT2] - Arrangements and Hypergeometric Integrals, in preparation.

[STV] V. Schechtman, H. Terao, and A. Varchenko, Cohomology of local systems and the KacKazhdan condition for singular vectors, J. Pure Appl. Algebra 100 (1995), 93-102.

[SV] V. Schechtman and A. Varchenko, Arrangements of hyperplanes and Lie algebra homology, Invent. Math. 106 (1991), 139-194.

[Va] A. Varchenko, Multidimensional Hypergeometric Functions and Representation Theory of Lie Algebras and Quantum Groups, Adv. Ser. Math. Phys., vol. 21, World Scientific, River Edge, NJ, 1995.

[Yu] S. Yuzvinsky, Cohomology of the Brieskorn-Orlik-Solomon algebras, Comm. Algebra 23 (1995), 5339-5354.

Department of Mathematics, Louisiana State University, Baton Rouge, LA 70803

E-mail address: cohen@math.lsu.edu

$U R L:$ http://www.math.1su.edu/ ${ }^{\text {cohen }}$

Department of Mathematics, University of Wisconsin, Madison, WI 53706

E-mail address: orlik@math.wisc.edu 